\title{
On eigenfunction expansions associated with wave propagation along ducts with wave-bearing boundaries
}

\author{
by \\ Jane B. Lawrie \\ School of IS, Computing and Mathematics \\ Mathematical Sciences \\ Brunel University, Uxbridge, Middlesex, UB8 3PH. \\ jane.lawrie@brunel.ac.uk
}

February 2007

\begin{abstract}
A class of boundary value problems, that has application in the propagation of waves along ducts in which the boundaries are wave-bearing, is considered. This class of problems is characterised by the presence of high order derivatives of the dependent variable(s) in the duct boundary conditions. It is demonstrated that the underlying eigenfunctions are linearly dependent and, most significantly, that an eigenfunction expansion representation of a suitably smooth function, say $f(y)$, converges point-wise to that function. Two physical examples are presented. It is demonstrated that, in both cases, the eigenfunction representation of the solution is rendered unique via the application of suitable edge conditions. Within the context of these two examples, a detailed discussion of the issue of completeness is presented.
\end{abstract}




\section{Introduction}

The class of boundary value problems considered in this article is one that occurs in a number of fields of applied mathematics, especially water-waves and structural acoustics. Common to both these areas are problems which involve the propagation of waves along waveguides in which one or both of the boundaries are described by the Robin's or a higher-order condition. It is with the high-order boundary conditions that this article, is concerned. That is, boundary conditions that contain high-order spacial derivatives in the axial direction of the waveguide.

Andronov and Belinsky (1990) were amongst the first authors to demonstrate that the eigenfunctions arising for problems of this class display unusual orthogonality properties. They considered a specific but typical example. Later, Lawrie and Abrahams (1999) described the general boundary value problem for a broad class of such problems. They studied the underlying eigensystem and derived the orthogonality property for the general case. They further demonstrated that a range of physical problems could be successfully solved by expanding, for example, the velocity potential in terms of these eigenfunctions. Since then several authors, see for example Warren et al (2002), Evans and Porter (2003), Kaplunov et al (2004), Lawrie and Guled (2006) have used this class of eigenfunction expansion together with the appropriate orthogonality property to solve a wide range of problems. To date, however, to the best of the author's knowledge, little has been published regarding the formal properties of the eigenfunctions. This article goes some way to redressing the balance by establishing, in a rigorous manner, some of the most significant properties of the eigenfunctions for this class of problem.

In section 2 the general boundary value problem is stated and the orthogonality property is presented in a slightly different form to that derived by Lawrie and Abrahams (1999). The derivation of the recast form is given in Appendix A. The analytic properties of the eigenfunctions are presented in section 3. Firstly, it is proved that the infinite set of eigenfunctions is linearly dependent. Most significantly, however, it is proved that an eigenfunction expansion representation of a suitably smooth function, say $f(y)$, converges point-wise to that function. Crucial to these results is the evaluation of several infinite sums. These are evaluated using a complex variable method, details of which may be found in Lawrie and Kirby (2006). A comprehensive discussion of the properties proved in section 3 and their implications is presented in section 4 . The most pertinent points, for 
example completeness of the eigenfunctions and uniqueness of the eigenfunction expansion, are demonstrated by use of two physical examples. Some infinite sums relevant to this section are discussed in Appendix B. Finally, some concluding remarks are made in section 5.

\section{The generalised boundary value problem}

The analysis presented herein applies equally to boundary value problems governed by Laplace's or Helmholtz' equation. For ease of exposition, however, the general boundary value problem is posed in terms of Helmholtz' equation, that is

$$
\left\{\frac{\partial^{2}}{\partial x^{2}}+\frac{\partial^{2}}{\partial y^{2}}+1\right\} \phi(x, y)=0
$$

in which $x$ and $y$ are the usual Cartesian coordinates but are non-dimensionalised with respect to $k^{-1}$. Here $k$ is the fluid wavenumber, that is $k=\omega / c$ where $c$ is the fluid sound speed and $\omega$ is the frequency of the harmonic time dependence. The field equation holds in a strip of finite height $0 \leq y \leq a$ and infinite length $-\infty<x<\infty$ which is bounded by walls described by high-order boundary conditions. The mathematical statement of the boundary conditions is

$$
\mathcal{L}_{a}\left(\frac{\partial}{\partial x}\right) \frac{\partial \phi}{\partial y}+\mathcal{M}_{a}\left(\frac{\partial}{\partial x}\right) \phi=0, \quad y=a, \quad-\infty<x<\infty
$$

on the upper waveguide surface together with

$$
\mathcal{L}_{0}\left(\frac{\partial}{\partial x}\right) \frac{\partial \phi}{\partial y}+\mathcal{M}_{0}\left(\frac{\partial}{\partial x}\right) \phi=0, \quad y=0, \quad-\infty<x<\infty
$$

on the lower surface. Here $\mathcal{L}_{p}\left(\frac{\partial}{\partial x}\right)$ and $\mathcal{M}_{p}\left(\frac{\partial}{\partial x}\right), p=a, 0$, are differential operators of the form

$$
\mathcal{L}_{p}\left(\frac{\partial}{\partial x}\right)=\sum_{k=0}^{K_{p}} c_{k}^{p} \frac{\partial^{2 k}}{\partial x^{2 k}}, \quad \mathcal{M}_{p}\left(\frac{\partial}{\partial x}\right)=\sum_{j=0}^{J_{p}} d_{j}^{p} \frac{\partial^{2 j}}{\partial x^{2 j}},
$$

where $c_{k}^{0}, c_{k}^{a}, d_{j}^{0}, d_{j}^{a}$ are constant coefficients. Note that, only even derivatives in $x$ are included in the above boundary conditions. (Odd derivatives do occur in systems which are damped, and the presence of such derivatives significantly alters the nature of the underlying eigensystem. In particular, the dispersion relation will not be an even function of the wave number. It is not, therefore, immediately obvious that the results presented herein apply to such systems.) Note also, that higher derivatives in $y$ are easily removed by recourse to equation (2.1), hence the absence of such terms in (2.2) and (2.3). 
The general solution of the boundary value problem described by (2.1)-(2.4) can be expressed as a separable eigenfunction expansion of the form

$$
\phi(x, y)=\sum_{n=0}^{\infty} A_{n} Y_{n}(y) e^{ \pm i s_{n} x}, \quad x \gtrless 0 .
$$

Here $Y_{n}(y)$ satisfies the eigensystem

$$
Y_{n}^{\prime \prime}(y)=\gamma_{n}^{2} Y_{n}(y), \quad \gamma_{n}=\left(s_{n}^{2}-1\right)^{1 / 2}
$$

where the primes denote differentiation with respect to $y$ and the quantities $s_{n}, n=$ $0,1,2, \ldots$ are defined as the roots of the coupled equations

$$
\begin{aligned}
P_{a}\left(s_{n}\right) Y_{n}^{\prime}(a)+Q_{a}\left(s_{n}\right) Y_{n}(a) & =0 \\
P_{0}\left(s_{n}\right) Y_{n}^{\prime}(0)+Q_{0}\left(s_{n}\right) Y_{n}(0) & =0 .
\end{aligned}
$$

The functions $P_{p}(s)$ and $Q_{p}(s), p=a, 0$, are characteristic polynomials and correspond to the action of the operators $\mathcal{L}_{p}\left(\frac{\partial}{\partial x}\right)$ and $\mathcal{M}_{p}\left(\frac{\partial}{\partial x}\right)$ on the eigen-expansion $(2.5)$, i.e. $P_{p}\left(s_{n}\right) \equiv$ $\mathcal{L}_{p}\left(i s_{n}\right), Q_{p}\left(s_{n}\right) \equiv \mathcal{M}_{p}\left(i s_{n}\right)$.

It is a simple matter to show that (2.6)-(2.8) imply an explicit form for $Y_{n}(y)$ which without loss of generality may be written as:

$$
Y_{n}(y)=P_{0}\left(s_{n}\right) \cosh \left(\gamma_{n} y\right)-\frac{1}{\gamma_{n}} Q_{0}\left(s_{n}\right) \sinh \left(\gamma_{n} y\right)
$$

The dispersion relation can be deduced using (2.9) together with (2.7). That is

$$
\begin{aligned}
K(s) & =\left[\gamma^{2} P_{0}(s) P_{a}(s)-Q_{0}(s) Q_{a}(s)\right] \frac{\sinh (\gamma a)}{\gamma}+\left[Q_{a}(s) P_{0}(s)-P_{a}(s) Q_{0}(s)\right] \cosh (\gamma a) \\
& =\tau(s) \frac{\sinh (\gamma a)}{\gamma}+\lambda(s) \cosh (\gamma a)=0,
\end{aligned}
$$

where $\gamma^{2}=s^{2}-1$. The fact that the operators (2.4) contain only even derivatives in $x$ ensures that the characteristic polynomials are functions of $s^{2}$. It is straightforward to prove that, for the general case in which $P_{p}(s), Q_{p}(s), p=0, a$, contain real coefficients:

(i) for every root $s_{n}$ there is another root $-s_{n}$;

(ii) there is a finite number of real roots (that number depending on the number of real zeros of the polynomials $\tau(s)$ and $\lambda(s)$ ) located on $|s|>1$;

(iii) there is an infinite number of roots located on the imaginary axis of $\gamma$, or equivalently a finite number on $|\Re(s)|<1, \Im(s)=0$, and an infinite number on $\Re(s)=0, \Im(s)>0$; 
(iv) there is a finite number of roots, $s_{n}$, with non-zero real and imaginary parts.

In order that (2.5) converge for the $x \gtrless 0$, the convention is adopted that the $+s_{n}$ roots have either $\Re\left(s_{n}\right)>0, \Im\left(s_{n}\right)=0$ or $\Im\left(s_{n}\right)>0$. They are ordered sequentially, real roots first, starting with the largest real root and then by increasing imaginary part. Thus, $s_{0}$ is always the largest real root. It is worthwhile commenting that for any complex root, say $s_{c}$, lying in the upper half of the complex $s$-plane, then $-s_{c}^{*}$, where * indicates the complex conjugate, also lies in this half plane. Further, it is assumed that $s_{n} \neq 0$ and that no root is repeated.

It is expedient to recast the orthogonality relation presented by Lawrie and Abrahams (1999) into a slightly different form. The new form is equivalent to the original, but more convenient for the analysis that follows. Thus, the eigenfunctions $Y_{n}(y)$ described by (2.6)(2.8) have the orthogonality property

$$
\begin{aligned}
\int_{0}^{a} Y_{m} Y_{n} d y & +\frac{2 p_{m n}}{\left(t_{m n}+t_{n m}\right)}\left(Y_{m}^{\prime}(a) Y_{n}^{\prime}(a)-Y_{m}^{\prime}(0) Y_{n}^{\prime}(0)\right) \\
& +\frac{2 q_{m n}}{\left(t_{m n}+t_{n m}\right)}\left(Y_{m}(a) Y_{n}(a)-Y_{m}(0) Y_{n}(0)\right) \\
& +\frac{r_{m n}}{\left(t_{m n}+t_{n m}\right)}\left(Y_{m}(a) Y_{n}^{\prime}(a)+Y_{n}(a) Y_{m}^{\prime}(a)\right) \\
& -\frac{r_{m n}}{\left(t_{m n}+t_{n m}\right)}\left(Y_{m}(0) Y_{n}^{\prime}(0)+Y_{n}(0) Y_{m}^{\prime}(0)\right)=\delta_{m n} E_{n}
\end{aligned}
$$

where

$$
\begin{aligned}
t_{m n} & =P_{0}\left(s_{m}\right) Q_{a}\left(s_{n}\right)-P_{a}\left(s_{m}\right) Q_{0}\left(s_{n}\right), \\
p_{m n} & =\frac{P_{0}\left(s_{m}\right) P_{a}\left(s_{n}\right)-P_{0}\left(s_{n}\right) P_{a}\left(s_{m}\right)}{\gamma_{m}^{2}-\gamma_{n}^{2}}, \\
q_{m n} & =\frac{Q_{0}\left(s_{m}\right) Q_{a}\left(s_{n}\right)-Q_{0}\left(s_{n}\right) Q_{a}\left(s_{m}\right)}{\gamma_{m}^{2}-\gamma_{n}^{2}}, \\
r_{m n} & =\frac{t_{m n}-t_{n m}}{\gamma_{m}^{2}-\gamma_{n}^{2}}
\end{aligned}
$$

and the non-zero constant $E_{n}$ is given by

$$
E_{n}=\frac{Y_{n}(a)}{2 s_{n} P_{a}\left(s_{n}\right)} K^{\prime}\left(s_{n}\right) .
$$

Note that, despite the fact that there may be a finite number of complex eigenvalues, it is chosen to cast the orthogonality relation in terms of $Y_{n}(y)$ and $Y_{m}(y)$ rather than the more conventional formulation which would employ $Y_{n}(y)$ and $Y_{m}^{*}(y)$. The reason for this becomes apparent in section 4. Expression (2.11) has the advantage that the denominator $t_{m n}+t_{n m} \neq 0$, however, this orthogonality property can be recast into various other forms. 
If, for example, $Q_{p}(s) \neq 0, p=0, a$ then it is convenient to eliminate $Y_{n(m)}(a)$ and $Y_{n(m)}(0)$ by using (2.7) and (2.8). It is then found that

$$
\begin{aligned}
\int_{0}^{a} Y_{m} Y_{n} d y & +\left\{\frac{P_{a}\left(s_{n}\right) Q_{a}\left(s_{m}\right)-P_{a}\left(s_{m}\right) Q_{a}\left(s_{n}\right)}{\left(\gamma_{m}^{2}-\gamma_{n}^{2}\right)}\right\} \frac{Y_{m}^{\prime}(a) Y_{n}^{\prime}(a)}{Q_{a}\left(s_{m}\right) Q_{a}\left(s_{n}\right)} \\
& -\left\{\frac{P_{0}\left(s_{n}\right) Q_{0}\left(s_{m}\right)-P_{0}\left(s_{m}\right) Q_{0}\left(s_{n}\right)}{\left(\gamma_{m}^{2}-\gamma_{n}^{2}\right)}\right\} \frac{Y_{m}^{\prime}(0) Y_{n}^{\prime}(0)}{Q_{0}\left(s_{m}\right) Q_{0}\left(s_{n}\right)}=\delta_{m n} E_{n} .
\end{aligned}
$$

The quantity $P_{p}\left(s_{j}\right) Q_{p}\left(s_{n}\right)-P_{p}\left(s_{n}\right) Q_{p}\left(s_{j}\right), p=0, a$ is a polynomial in the two variables $\gamma_{n}^{2}$ and $\gamma_{j}^{2}$ and is divisible by $\gamma_{n}^{2}-\gamma_{j}^{2}$. It follows that

$$
\frac{P_{p}\left(s_{n}\right) Q_{p}\left(s_{m}\right)-P_{p}\left(s_{m}\right) Q_{p}\left(s_{n}\right)}{\left(\gamma_{m}^{2}-\gamma_{n}^{2}\right)}=\sum_{j=0}^{N_{p}} \sum_{k=0}^{N_{p}} b_{j k}^{p} \gamma_{m}^{2 j} \gamma_{n}^{2 k}, \quad p=0, a
$$

where the coefficients $b_{j k}^{p}=b_{k j}^{p}$ are related to $c_{k}^{p}$ and $d_{j}^{p}$ of $(2.4)$ and $N_{p}=\max \left\{J_{p}-1, K_{p}-\right.$ $1\}, p=0, a$. It follows that

$$
\begin{aligned}
\int_{0}^{a} Y_{m} Y_{n} d y & +\sum_{j=0}^{N_{a}} \sum_{k=0}^{N_{a}} b_{j k}^{a} \gamma_{m}^{2 j} \gamma_{n}^{2 k} \frac{Y_{m}^{\prime}(a) Y_{n}^{\prime}(a)}{Q_{a}\left(s_{m}\right) Q_{a}\left(s_{n}\right)} \\
& -\sum_{j=0}^{N_{0}} \sum_{k=0}^{N_{0}} b_{j k}^{0} \gamma_{m}^{2 j} \gamma_{n}^{2 k} \frac{Y_{m}^{\prime}(0) Y_{n}^{\prime}(0)}{Q_{0}\left(s_{m}\right) Q_{0}\left(s_{n}\right)}=\delta_{m n} E_{n} .
\end{aligned}
$$

Should either $Q_{a}(s)=0$ or $Q_{0}(s)=0$, then (2.11) can be arranged into a form analogous to $(2.19)$ by eliminating $Y_{n(m)}^{\prime}(a)$ and $Y_{n(m)}^{\prime}(0)$ (as opposed to $Y_{n(m)}(a)$ and $\left.Y_{n(m)}(0)\right)$ using (2.7) and (2.8).

In this article attention is restricted to the following situations:

i) $Q_{p}(s) \neq 0, \quad\left|\frac{P_{p}(s)}{Q_{p}(s)}\right|=O\left(s^{2\left(K_{p}-J_{p}\right)}\right)$ as $|s| \rightarrow \infty$, with $K_{p}>J_{p}, p=0, a$;

ii) $Q_{0}(s)=0, \quad P_{0}(s) \neq 0, \quad\left|\frac{P_{a}(s)}{Q_{a}(s)}\right|=O\left(s^{2\left(K_{a}-J_{a}\right)}\right)$ as $|s| \rightarrow \infty \quad$ with $K_{a}>J_{a}$;

iii) $Q_{a}(s)=0, \quad P_{a}(s) \neq 0 \quad\left|\frac{P_{0}(s)}{Q_{0}(s)}\right|=O\left(s^{2\left(K_{0}-J_{0}\right)}\right)$ as $|s| \rightarrow \infty$ with $K_{0}>J_{0}$.

Since for each of these cases $K_{p}>J_{p}, p=0, a$, the quantity $N_{p}$ is henceforth defined by $N_{p}=K_{p}-1, p=0, a$. It is straightforward then to show that

$$
\gamma_{n+K_{a}+K_{0}} \sim \frac{i n \pi}{a}-\frac{i n \pi \lambda(i n \pi / a)}{a^{2} \tau(i n \pi / a)}, \quad n>>1
$$

where $\gamma_{n}=\left(s_{n}^{2}-1\right)^{1 / 2}$ and $s_{n}, n=0,1,2, \ldots$ are the roots to (2.10). Since condition i), ii) or iii) applies, $|\tau(s)|>|\gamma \lambda(s)|$ as $|s| \rightarrow \infty$. Thus, the second term on the right hand side 
of $(2.20)$ is $o\left(n^{-1}\right)$ as $n \rightarrow \infty$. The reason for the shift in counter seen in (2.20) becomes apparent in section 4 . It should be noted that (2.5) reduces to the usual Fourier cosine series if $Q_{p}(s)=0, p=0, a$ and $P_{p}(s), p=0, a$ is constant whilst the standard SturmLiouville case is retrieved if $P_{p}(s)$ and $Q_{p}(s)$ are all constant, $p=0, a$. There are many excellent texts that deal with analytic properties of these series, see for example Hanna and Rowland (1990), this article is concerned only with the situation in which one or both boundary conditions contain high-order derivatives. Excluded from this study are the cases $P_{0}(s)=0$ or $P_{a}(s)=0$ for which the eigenvalues $\gamma_{n}$ do not have the form (2.20).

\section{Analytic properties of the eigenfunctions}

\section{Theorem 1}

Given that $P_{p}(s)$ and $Q_{p}(s), p=0$, a satisfy condition $\left.i\right)$, ii) or iii) outlined above, the eigenfunctions $Y_{n}(y)$ described by (2.6)-(2.8) are linearly dependent for $0 \leq y \leq a$. Furthermore, there are exactly $K_{0}+K_{a}$ linear combinations of the eigenfunctions that are equal to zero. These linear combinations have the form

$$
\sum_{n=0}^{\infty} \frac{Y_{n}(a) Y_{n}(y) \gamma_{n}^{2 q}}{P_{a}\left(s_{n}\right) E_{n}}=0, \quad 0 \leq y \leq a, \quad q=0,1,2, \ldots K_{a}-1, \quad K_{a}>0
$$

and

$$
\sum_{n=0}^{\infty} \frac{Y_{n}(y) \gamma_{n}^{2 q}}{E_{n}}=0, \quad 0 \leq y \leq a, \quad q=0,1,2, \ldots K_{0}-1, \quad K_{0}>0
$$

\section{Proof}

Consider first expression (3.1). This sum can be evaluated by analysing the families of poles in the integrand of a suitably chosen integral. The method is the same as that used by Lawrie and Kirby (2006). The appropriate integral is

$$
I_{1}(y)=\frac{1}{2 \pi i} \int_{-\infty}^{\infty} \frac{s Y(s, y) f(s)}{K(s)} d s, \quad 0 \leq y \leq a,
$$

where the path of integration is indented above(below) any poles on the negative(positive) real axis. The function $Y(s, y)$ is defined by

$$
Y(s, y)=P_{0}(s) \cosh (\gamma y)-\frac{1}{\gamma} Q_{0}(s) \sinh (\gamma y)
$$

where $\gamma=\left(s^{2}-1\right)^{1 / 2}$. Thus, the eigenfunctions $Y_{n}(y)$ are expressed in terms of $Y(s, y)$ by $Y_{n}(y)=Y\left(s_{n}, y\right), n=0,1,2 \ldots$ Note that, since the integrand is odd, $I_{1}(y)=0$. The 
function $f(s)$ is any even, analytic function which, in order that the integrand converge when $y=a$, satisfies $|f(s)| \leq M s^{2 N_{a}}$ as $|s| \rightarrow \infty$ where $M$ is an arbitrary constant. Thus, by the extended form of Liouville's theorem, see Noble (1988), $f(s)$ must be a polynomial of, at most, degree $2 N_{a}$. Clearly the integrand has poles only when $K(s)=0$. On deforming the path of integration onto a semi-circular arc of radius $R>>1$ in the upper half plane and evaluating the residue contributions for the poles crossed, it is found that, as $R \rightarrow \infty$

$$
\sum_{n=0}^{\infty} \frac{s_{n} Y_{n}(y) f\left(s_{n}\right)}{K^{\prime}\left(s_{n}\right)}=\sum_{n=0}^{\infty} \frac{Y_{n}(y) Y_{n}(a) f\left(s_{n}\right)}{P_{a}\left(s_{n}\right) E_{n}}=0 .
$$

Since $f(s)$ is an even polynomial it may be cast in terms of $\gamma^{2}=s^{2}-1$ and (3.1) immediately follows. There are exactly $N_{a}+1=K_{a}$ sums of this form obtained by choosing $q=$ $0,1, \ldots N_{a}$ in (3.1). Sums involving more general polynomials, $f\left(s_{n}\right)$, are simply linear combinations of these basic forms. Note that this result applies only for the situation $N_{a} \geq 0$, equivalently $K_{a} \geq 1$ since the integral $I_{1}(y)$ does not converge for $K_{a}=0$.

Expression (3.2) is proved using the same technique but with the integral

$$
I_{2}(y)=\frac{1}{2 \pi i} \int_{-\infty}^{\infty} \frac{s Y(s, y) P_{a}(s) f(s)}{K(s) Y(s, a)} d s, \quad 0 \leq y \leq a,
$$

in which, unlike (3.3), the integrand contains two infinite families of poles. It is found that

$$
\sum_{n=0}^{\infty} \frac{Y_{n}(y) f\left(s_{n}\right)}{E_{n}}+2 \sum_{n=0}^{\infty} \frac{d_{n} Y\left(d_{n}, y\right) f\left(d_{n}\right) P_{a}\left(d_{n}\right)}{\left.K\left(d_{n}\right) \frac{d}{d s} Y(s, a)\right|_{s=d_{n}}}=0
$$

where $d_{n}, n=0,1,2, \ldots$ are the roots of $Y(s, a)=0$. It is convenient to rearrange the second sum of (3.7) using the relation $\delta_{n} P_{0}\left(d_{n}\right)=Q_{0}\left(d_{n}\right) \tanh \left(\delta_{n} a\right)$ which, with $\delta_{n}=\left(d_{n}^{2}-1\right)^{1 / 2}$, is a direct consequence of $Y\left(d_{n}, a\right)=0$. Thus,

$$
K\left(d_{n}\right)=-\frac{P_{a}\left(d_{n}\right) Q_{0}\left(d_{n}\right)}{\cosh \left(\delta_{n} a\right)}
$$

and

$$
Y\left(d_{n}, y\right)=-\frac{Q_{0}\left(d_{n}\right) \sinh \left[\delta_{n}(y-a)\right]}{\delta_{n} \cosh \left(\delta_{n} a\right)}
$$

so that (3.7) becomes

$$
\sum_{n=0}^{\infty} \frac{Y_{n}(y) f\left(s_{n}\right)}{E_{n}}+2 \sum_{n=0}^{\infty} \frac{d_{n} \sinh \left[\delta_{n}(y-a)\right] f\left(d_{n}\right)}{\left.\delta_{n} \frac{d}{d s} Y(s, a)\right|_{s=d_{n}}}=0 .
$$

The second sum can be evaluated by analysing the pole structure of the integrand of $I_{3}(y)$ where

$$
I_{3}(y)=\frac{1}{2 \pi i} \int_{-\infty}^{\infty} \frac{s \sinh [\gamma(y-a)] f(s)}{\gamma Y(s, a)} d s, \quad 0 \leq y \leq a
$$


and it is found that

$$
\sum_{n=0}^{\infty} \frac{d_{n} \sinh \left[\delta_{n}(y-a)\right] f\left(d_{n}\right)}{\left.\delta_{n} \frac{d}{d s} Y(s, a)\right|_{s=d_{n}}}=0 .
$$

As for the previous sum, $f(s)$ is an even polynomial, in this case of degree, at most, $2 N_{0}$, and therefore (3.2) follows. There are exactly $N_{0}+1=K_{0}$ sums of this form obtained by choosing $q=0,1, \ldots N_{0}$ in (3.2). This result applies only for the situation $N_{0} \geq 0$, equivalently $K_{0} \geq 1$ since the integral $I_{2}(y)$ does not converge for $K_{0}=0$.

\section{Lemma}

Given that $P_{p}(s)$ and $Q_{p}(s), p=0$, a satisfy condition $\left.i\right)$, ii) or iii) outlined above, the eigenfunctions $Y_{n}(y)$ described by (2.6)-(2.8) have the property

$$
\sum_{n=0}^{\infty} \frac{Y_{n}(y) Y_{n}(v)}{E_{n}}=\delta(v-y)+\delta(v+y)+\delta(v+y-2 a), \quad 0 \leq v \leq a, \quad 0 \leq y \leq a .
$$

\section{Proof}

In order to prove this result, it is expedient first to evaluate the convergent sum

$$
\sum_{n=0}^{\infty} \frac{Y_{n}(y) Z_{n}(v)}{E_{n}}
$$

where

$$
Z_{n}(v)=\int^{v} Y_{n}(u) d u
$$

This sum can be evaluated by analysing the families of poles of the integral $I_{4}(v, y)$ where

$$
I_{4}(v, y)=\frac{1}{2 \pi i} \int_{-\infty}^{\infty} \frac{s Y(s, y) Z(s, v) P_{a}(s)}{K(s) Y(s, a)} d s, \quad-2 a<v+y \leq 2 a .
$$

Here

$$
Z(s, v)=\int^{v} Y(s, u) d u=P_{0}(s) \frac{\sinh (\gamma v)}{\gamma}-Q_{0}(s) \frac{\cosh (\gamma v)}{\gamma^{2}}
$$

and the path of integration is indented above(below) and poles on the negative(positive) real axis. Since the integrand is odd, $I_{4}(v, y)=0$.

The integrand has poles when: (i) $K(s)=0$ i.e $s=s_{n}, n=0,1,2 \ldots$; (ii) $Y(s, a)=0$ i.e. $s=d_{n}, n=0,1,2 \ldots$; (iii) $s=1$. Thus, on deforming the path of integration onto a semi-circular arc of radius $R>>1$ in the upper half plane and evaluating the residue contributions for the poles crossed, it is found that, as $R \rightarrow \infty$

$$
\begin{aligned}
\sum_{n=0}^{\infty} \frac{s_{n} Y_{n}(y) Z_{n}(v) P_{a}\left(s_{n}\right)}{K^{\prime}\left(s_{n}\right) Y_{n}(a)} & +\sum_{n=0}^{\infty} \frac{d_{n} Y\left(d_{n}, y\right) Z\left(d_{n}, v\right)}{\left.K\left(d_{n}\right) \frac{d}{d s} Y(s, a)\right|_{s=d_{n}}} \\
& -\frac{Q_{0}(1) P_{a}(1)\left\{P_{0}(1)-y Q_{0}(1)\right\}}{K(1)\left\{P_{0}(1)-a Q_{0}(1)\right\}}=2 H(v+y-2 a),
\end{aligned}
$$


where $H($.$) is the Heaviside function, defined by$

$$
H(x)=\left\{\begin{array}{cc}
1, & x>0 \\
\frac{1}{2}, & x=0 \\
0, & x<0
\end{array} .\right.
$$

The Heaviside function of (3.18) arises due to the contribution from the semi-circular arc when $v=y=a$.

On using (2.16), it is found that,

$$
\begin{aligned}
\sum_{n=0}^{\infty} \frac{Y_{n}(y) Z_{n}(v)}{E_{n}} & =2 H(v+y-2 a)+\frac{Q_{0}(1) P_{a}(1)\left\{P_{0}(1)-y Q_{0}(1)\right\}}{K(1)\left\{P_{0}(1)-a Q_{0}(1)\right\}} \\
& -2 \sum_{n=0}^{\infty} \frac{d_{n} Y\left(d_{n}, y\right) Z\left(d_{n}, v\right)}{\left.K\left(d_{n}\right) \frac{d}{d s} Y(s, a)\right|_{s=d_{n}}}
\end{aligned}
$$

It is convenient to rearrange the last term of (3.20) using (3.8), (3.9) and

$$
Z\left(d_{n}, v\right)=-\frac{Q_{0}\left(d_{n}\right) \cosh \left[\delta_{n}(v-a)\right]}{\delta_{n}^{2} \cosh \left(\delta_{n} a\right)}
$$

so that (3.20) becomes

$$
\begin{aligned}
\sum_{n=0}^{\infty} \frac{Y_{n}(y) Z_{n}(v)}{E_{n}} & =2 H(v+y-2 a)+\frac{Q_{0}(1) P_{a}(1)\left\{P_{0}(1)-y Q_{0}(1)\right\}}{K(1)\left\{P_{0}(1)-a Q_{0}(1)\right\}} \\
& +2 \sum_{n=0}^{\infty} \frac{d_{n} P_{0}\left(d_{n}\right) \sinh \left[\delta_{n}(y-a)\right] \cosh \left[\delta_{n}(v-a)\right]}{\left.\left(d_{n}^{2}-1\right) \sinh \left(\delta_{n} a\right) \frac{d}{d s} Y(s, a)\right|_{s=d_{n}}}
\end{aligned}
$$

The sum on the right hand side of (3.22) is again evaluated by analysing the pole structure of a suitable integral, in this case $I_{5}(v, y)$ where

$$
I_{5}(v, y)=-\frac{1}{2 \pi i} \int_{-\infty}^{\infty} \frac{s P_{0}(s) \sinh [\gamma(y-a)] \cosh [\gamma(v-a)]}{\left(s^{2}-1\right) \sinh (\gamma a) Y(s, a)} d s .
$$

As before, the path of integration is indented above(below) any poles on the negative(positive) real axis and, since the integrand is odd, $I_{5}(v, y)=0$. The integrand of $I_{5}(v, y)$ has poles when: (i) $\sinh (\gamma a)=0$ i.e $s=\left(1-n^{2} \pi^{2} / a^{2}\right)^{1 / 2}, n=0,1,2 \ldots$; (ii) $Y(s, a)=0$ i.e. $s=d_{n}$, $n=0,1,2 \ldots$; (iii) $s=1$. Again, on deforming the path of integration and evaluating the residue contribution for each pole, it is found that

$$
\begin{aligned}
2 \sum_{n=0}^{\infty} \frac{d_{n} P_{0}\left(d_{n}\right) \sinh \left[\delta_{n}(y-a)\right] \cosh \left[\delta_{n}(v-a)\right]}{\left.\left(d_{n}^{2}-1\right) \sinh \left(\delta_{n} a\right) \frac{d}{d s} Y(s, a)\right|_{s=d_{n}}} & =2 H(-v-y)+\frac{P_{0}(1)(a-y)}{a\left\{P_{0}(1)-a Q_{0}(1)\right\}} \\
& -\frac{2}{\pi} \sum_{n=1}^{\infty} \frac{1}{n} \sin \left(\frac{n \pi y}{a}\right) \cos \left(\frac{n \pi v}{a}\right)
\end{aligned}
$$


where the Heaviside function arises due to the contribution from the semi-circular arc for $v=y=0$. The sum on the right hand side of (3.24) is a discontinuous function of $y$ and $v$, which takes the value zero when $y=v=0$ or $y=v=a$. On using Gradshetyn and Rhyzik (1980), it is easily shown that for $-2 a<y+v<4 a$ and $0 \leq|v-y| \leq 2 a$

$$
\frac{2}{\pi} \sum_{n=1}^{\infty} \frac{1}{n} \sin \left(\frac{n \pi y}{a}\right) \cos \left(\frac{n \pi v}{a}\right)=-H(-y-v)-H(v-y)+H(y+v-2 a)+1-\frac{y}{a} .
$$

It follows from (3.22), $(3.24)^{1}$ and (3.25) that, for $0 \leq v \leq a$ and $0 \leq y \leq a$

$$
\begin{aligned}
\sum_{n=0}^{\infty} \frac{Y_{n}(y) Z_{n}(v)}{E_{n}} & =H(v-y)-H(-v-y)+H(v+y-2 a)+1-\frac{y}{a} \\
& +\frac{Q_{0}(1) P_{0}(1)\left\{P_{0}(1)-y Q_{0}(1)\right\}}{K(1)\left\{P_{0}(1)-a Q_{0}(1)\right\}}+\frac{P_{0}(1)(a-y)}{a\left\{P_{0}(1)-a Q_{0}(1)\right\}} .
\end{aligned}
$$

On differentiating (3.26) with respect to $v$ expression (3.13) immediately follows. Note that (3.26) and (3.13) hold only for $0 \leq v \leq a$ and $0 \leq y \leq a$.

\section{Theorem 2}

Given that the coefficients

$$
\begin{aligned}
A_{n} & =\frac{1}{E_{n}}\left\{\int_{0}^{a} f(v) Y_{n}(v) d v\right. \\
& +\sum_{m=0}^{\infty} \frac{2 A_{m} p_{m n}}{t_{m n}+t_{n m}}\left\{Y_{m}^{\prime}(a) Y_{n}^{\prime}(a)-Y_{m}^{\prime}(0) Y_{n}^{\prime}(0)\right\} \\
& +\sum_{m=0}^{\infty} \frac{2 A_{m} q_{m n}}{t_{m n}+t_{n m}}\left\{Y_{m}(a) Y_{n}(a)-Y_{m}(0) Y_{n}(0)\right\} \\
& +\sum_{m=0}^{\infty} \frac{2 A_{m} r_{m n}}{t_{m n}+t_{n m}}\left\{Y_{m}(a) Y_{n}^{\prime}(a)+Y_{m}^{\prime}(a) Y_{n}(a)\right\} \\
& \left.-\sum_{m=0}^{\infty} \frac{2 A_{m} r_{m n}}{t_{m n}+t_{n m}}\left\{Y_{m}(0) Y_{n}^{\prime}(0)-Y_{m}^{\prime}(0) Y_{n}(0)\right\}\right\}
\end{aligned}
$$

exist, where $f(y)$ is any function that is $M=\max \left\{2 K_{a}-1,2 K_{0}-1\right\}$ times differentiable on the domain $0 \leq y \leq a$ and the eigenfunctions $Y_{n}(y)$ are defined by (2.6)-(2.8), then, provided $P_{p}(s)$ and $Q_{p}(s), p=0$, a satisfy condition $\left.i\right)$, ii) or iii), the series

$$
\sum_{n=0}^{\infty} A_{n} Y_{n}(y)
$$

\footnotetext{
${ }^{1}$ The reader is advised that there are errors of sign in equations (3.24) and (3.26) as written above. These do not, however, affect the result (3.13). The correct forms for (3.24) and (3.26) are given at the end of this article
} 
converges point-wise to $f(y)$ for $0 \leq y \leq a$.

\section{Proof}

Assume that a suitably smooth function $f(y), 0 \leq y \leq a$ can be expressed as an eigenfunction expansion so that

$$
f(y)=\sum_{n=0}^{\infty} A_{n} Y_{n}(y) .
$$

Let $F_{N}(y)$ be the sum of the first $N$ terms of the eigenfunction expansion (3.27), thus

$$
F_{N}(y)=\sum_{n=0}^{N} A_{n} Y_{n}(y) .
$$

Without loss of generality, consider the case where $Q_{p}(s) \neq 0$ and $p=0$, a, then the coefficients $A_{n}$ can be recast as

$$
\begin{aligned}
A_{n}=\frac{1}{E_{n}}\left\{\int_{0}^{a} f(v) Y_{n}(v) d v\right. & +\sum_{m=0}^{\infty} A_{m} \sum_{j=0}^{N_{a}} \sum_{k=0}^{N_{a}} b_{j k}^{a} \gamma_{m}^{2 j} \gamma_{n}^{2 k} \frac{Y_{m}^{\prime}(a) Y_{n}^{\prime}(a)}{Q_{a}\left(s_{m}\right) Q_{a}\left(s_{n}\right)} \\
& \left.-\sum_{m=0}^{\infty} A_{m} \sum_{j=0}^{N_{0}} \sum_{k=0}^{N_{0}} b_{j k}^{0} \gamma_{m}^{2 j} \gamma_{n}^{2 k} \frac{Y_{m}^{\prime}(0) Y_{n}^{\prime}(0)}{Q_{0}\left(s_{m}\right) Q_{0}\left(s_{n}\right)}\right\}
\end{aligned}
$$

where $N_{p}=K_{p}-1, p=0, a$. This expression may be substituted into the eigenfunction expansion of the right hand side of (3.28), to obtain

$$
\begin{aligned}
\sum_{n=0}^{N} A_{n} Y_{n}(y) & =\sum_{n=0}^{N} \frac{Y_{n}(y)}{E_{n}}\left\{\int_{0}^{a} f(v) Y_{n}(v) d v\right. \\
& +\sum_{m=0}^{\infty} A_{m} \sum_{j=0}^{N_{a}} \sum_{k=0}^{N_{a}} b_{j k}^{a} \gamma_{m}^{2 j} \gamma_{n}^{2 k} \frac{Y_{m}^{\prime}(a) Y_{n}^{\prime}(a)}{Q_{a}\left(s_{m}\right) Q_{a}\left(s_{n}\right)} \\
& \left.-\sum_{m=0}^{\infty} A_{m} \sum_{j=0}^{N_{0}} \sum_{k=0}^{N_{0}} b_{j k}^{0} \gamma_{m}^{2 j} \gamma_{n}^{2 k} \frac{Y_{m}^{\prime}(0) Y_{n}^{\prime}(0)}{Q_{0}\left(s_{m}\right) Q_{0}\left(s_{n}\right)}\right\} .
\end{aligned}
$$

On interchanging the orders of summation and integration in the first term of (3.30), rearranging the subsequent terms using (2.7)-(2.8) and letting $N \rightarrow \infty$, expression (3.30) becomes

$$
\begin{aligned}
\sum_{n=0}^{\infty} A_{n} Y_{n}(y) & =\int_{0}^{a} f(v) \sum_{n=0}^{\infty} \frac{Y_{n}(y) Y_{n}(v)}{E_{n}} d v \\
& -\sum_{n=0}^{\infty} \frac{Y_{n}(y)}{E_{n}} \sum_{m=0}^{\infty} A_{m} \sum_{j=0}^{N_{a}} \sum_{k=0}^{N_{a}} b_{j k}^{a} \gamma_{m}^{2 j} \gamma_{n}^{2 k} \frac{Y_{m}^{\prime}(a) Y_{n}(a)}{Q_{a}\left(s_{m}\right) P_{a}\left(s_{n}\right)} \\
& -\sum_{n=0}^{\infty} \frac{Y_{n}(y)}{E_{n}} \sum_{m=0}^{\infty} A_{m} \sum_{j=0}^{N_{0}} \sum_{k=0}^{N_{0}} b_{j k}^{0} \gamma_{m}^{2 j} \gamma_{n}^{2 k} .
\end{aligned}
$$


Since the innermost two summations are finite and the summands are clearly separable in $m$ and $n$, this can be rewritten as

$$
\begin{aligned}
\sum_{n=0}^{\infty} A_{n} Y_{n}(y) & =\int_{-\infty}^{\infty} \mathcal{F}(v) \sum_{n=0}^{\infty} \frac{Y_{n}(y) Y_{n}(v)}{E_{n}} d v \\
& -\sum_{j=0}^{N_{a}} \sum_{k=0}^{N_{a}} b_{j k}^{a} \sum_{m=0}^{\infty} A_{m} \gamma_{m}^{2 j} \frac{Y_{m}^{\prime}(a)}{Q_{a}\left(s_{m}\right)} \sum_{n=0}^{\infty} \frac{Y_{n}(y) Y_{n}(a) \gamma_{n}^{2 k}}{E_{n} P_{a}\left(s_{n}\right)} \\
& -\sum_{j=0}^{N_{0}} \sum_{k=0}^{N_{0}} b_{j k}^{0} \sum_{m=0}^{\infty} A_{m} \gamma_{m}^{2 j} \sum_{n=0}^{\infty} \frac{Y_{n}(y) \gamma_{n}^{2 k}}{E_{n}}
\end{aligned}
$$

where

$$
\mathcal{F}(v)=f(v) H(v) H(a-v)
$$

Note that, due to the manner in which the Heaviside function is defined, this extension of $f(v)$ introduces a multiplicative half at the points $y=0$ and $y=a$. This, however, is consistent with the fact that two of the delta functions present in (3.13) pick up contributions from these points. Provided $N_{p} \geq 0$, the two inner sums are are zero, see (3.1) and (3.2). (The case $N_{p}=0$ corresponds to $P_{p}(s)=1$ which can occur only with $Q_{p}(s)=0, p=0, a$ and for which the coefficients $b_{j k}^{p}=0, p=0, a$.) Hence, on using Lemma 1, it is found that

$$
\sum_{n=0}^{\infty} A_{n} Y_{n}(y)=f(y), \quad 0 \leq y \leq a .
$$

\section{Discussion}

Theorem 1 proves that the eigenfunctions $Y_{n}(y), n=0,1,2, \ldots$ are linearly dependent and that there are exactly $K_{0}+K_{a}$ infinite, linear combinations of these functions that are equal to zero.

It is interesting, in this context, to note that, within any circular contour, $\Gamma$, of radius $R>>1, R>>s_{0}$ centered at the origin of the complex s-plane, the dispersion function $K(s)$, see $(2.10)$, has exactly $2\left(K_{0}+K_{a}\right)$ more roots than does $L(s)=\gamma \sinh (\gamma a), \gamma=$ $\left(s^{2}-1\right)^{1 / 2}$ which is the characteristic function that generates the standard Fourier cosine series. It follows that, corresponding to the zeros within $\Gamma$, there are exactly $K_{0}+K_{a}$ more eigenfunctions $Y_{n}(y)$ than there are for the standard cosine series. This result is proved using the Argument Principle, see for example, Churchill et al (1974). Since neither $K(s)$ or $L(s)$ have any poles and there are no repeated roots

$$
\int_{\Gamma}\left\{\frac{K^{\prime}(s)}{K(s)}-\frac{L^{\prime}(s)}{L(s)}\right\} d s=Z_{K}-Z_{L}
$$


where $Z_{K}$ and $Z_{L}$ are the number of zeros of $K(s)$ and $L(s)$ respectively within $\Gamma$. This integral is easily evaluated and, provided $R$ is sufficiently large, it is found that $Z_{K}-Z_{L}=$ $2\left(K_{0}+K_{a}\right)$. Note that this result explains the shift of counter seen in $(2.20)$.

A further interesting point is that, for problems involving elastic plates or membranes, there will always be exactly $K_{0}+K_{a}$ edge conditions to be applied at any plate/membrane edge. These give $K_{0}+K_{a}$ additional constraints on the solution to the boundary value problem described by (2.6)-(2.8).

The above three points indicate that $K_{0}+K_{a}$ of the eigenfunctions can be removed from the infinite set in order to produce a set of eigenfunctions that are linearly independent and span the space of functions that are $M=\max \left\{2 K_{a}-1,2 K_{0}-1\right\}$ differentiable on the domain $0 \leq y \leq a$. Equivalently, the solution obtained by application of the edge conditions is unique.

The statement of the Theorem 2 includes the assumption that the coefficients $A_{n}$ exist. If it can be proved that the eigenfunctions specified in Theorem 2 are complete, then it follows that the coefficients $A_{n}$ will exist. This is, however, not a trivial issue and is not addressed for the general case. It is clear, however, that for the many physical situations the coefficients $A_{n}$ do indeed exist even though completeness is, as yet, unproven. The conditions describing elastic plates and membranes are typical examples of physical boundaries conditions satisfying (2.7)-(2.8), and for these it is the case that $Q_{p}(s), p=0, a$ is constant. Under such circumstances (2.19) reduces to

$$
\begin{aligned}
\int_{0}^{a} Y_{m} Y_{n} d y & +\frac{1}{Q_{a}^{2}} \sum_{j=0}^{N_{a}-1} \sum_{k=0}^{N_{a}-1} b_{j k}^{a} Y_{m}^{(2 j+1)}(a) Y_{n}^{(2 k+1)}(a) \\
& -\frac{1}{Q_{0}^{2}} \sum_{j=0}^{N_{0}-1} \sum_{k=0}^{N_{0}-1} b_{j k}^{0} Y_{m}^{(2 j+1)}(0) Y_{n}^{(2 k+1)}(0)=\delta_{m n} E_{n}
\end{aligned}
$$

where $Q_{p}, p=0, a$ are constants and the bracketed superscripts indicate the order of a derivative. Even though many such orthogonality relations are not inner products in the strict sense (since $\left(Y_{n}, Y_{n}\right) \ngtr 0$ ), it is convenient to use an inner product notation to represent them. Thus, (4.2) is rewritten as

$$
\left(Y_{m}, Y_{m}\right)=\delta_{m n} E_{n}
$$


where

$$
\begin{aligned}
\left(Y_{m}, Y_{m}\right)=\int_{0}^{a} Y_{m} Y_{n} d y & +\frac{1}{Q_{a}^{2}} \sum_{j=0}^{N_{a}-1} \sum_{k=0}^{N_{a}-1} b_{j k}^{a} Y_{m}^{(2 j+1)}(a) Y_{n}^{(2 k+1)}(a) \\
& -\frac{1}{Q_{0}^{2}} \sum_{j=0}^{N_{0}-1} \sum_{k=0}^{N_{0}-1} b_{j k}^{0} Y_{m}^{(2 j+1)}(0) Y_{n}^{(2 k+1)}(0) .
\end{aligned}
$$

Then the coefficients $A_{n}$ assume the form

$$
A_{n}=\frac{\left(f, Y_{n}\right)}{\left(Y_{n}, Y_{n}\right)}
$$

which is clearly defined and exists for all suitably smooth functions, $f(y)$. It is important to note that $\left(Y_{n}, Y_{n}\right) \neq 0$ whereas the quantity $\left(Y_{n}, Y_{n}^{*}\right)$ will be zero if $s_{n}$ is complex. It is for this reason that the orthogonality relation is formulated in terms of $Y_{n}(y)$ and $Y_{m}(y)$ rather than $Y_{n}(y)$ and $Y_{m}^{*}(y)$.

It is useful to consider two specific examples. In both cases, (non-dimensional) harmonic time dependence $e^{-i t}$ is implicitly assumed. The first example is one for which it is a relatively straightforward procedure to confirm that the eigenfunctions are complete, whilst for the second it is not. The purpose of the first example is to demonstrate that, even though the eigenfunctions are linearly dependent, a unique eigenfunction expansion representation of the solution to a given physical problem can be constructed by application of an appropriate number of physically relevant edge conditions. The uniqueness of this solution is not in doubt since this example belongs to a class of problem that can also be solved via Fourier integral methods, see Lawrie and Abrahams (1999). The second example cannot be solved by recourse to Fourier integrals. In this case a viable solution method is to represent the fluid velocity as an eigenfunction expansion and use the appropriate orthogonality property together with the edge conditions. Since the coefficients of the eigenfunction expansion can be explicitly evaluated, Theorem 2 guarantees point-wise convergence to the solution.

\subsection{Example 1}

Firstly, consider a waveguide in which both the upper an lower surfaces comprise membranes but with different masses per unit area and/or tension. Then, $P_{0}(s)=-s^{2}+\mu_{1}^{2}, Q_{0}=-\alpha_{1}$, $P_{a}(s)=-s^{2}+\mu_{2}^{2}$ and $Q_{a}=\alpha_{2}$ where the definitions of the constants $\alpha_{1}, \alpha_{2}, \mu_{1}, \mu_{2}$ are given, for example, by Warrenet al (2002). In this case, the dispersion relation has no complex roots, they are all either strictly real or imaginary. Expression (4.3) holds, together with

$$
\left(Y_{m}, Y_{m}\right)=\int_{0}^{a} Y_{m} Y_{n} d y+\frac{1}{\alpha_{2}} Y_{m}^{\prime}(a) Y_{n}^{\prime}(a)+\frac{1}{\alpha_{1}} Y_{m}^{\prime}(0) Y_{n}^{\prime}(0)
$$


which is, in fact, a true inner product. Various forms of this orthogonality relation have appeared in the literature, see Lawrie and Abrahams (1999), Warren et al (2002), Kaplunov et al (2004) and Lawrie and Guled (2006). The norm for the inner product (4.6) is defined by

$$
\|f\|^{2}=(f, f) \geq 0
$$

It follows that completeness is proved for this particular set of eigenfunctions, $Y_{n}(y), n=$ $0,1,2, \ldots$, if it can be shown that

$$
\left\|f(y)-F_{n}(y)\right\| \rightarrow 0 \quad \text { as } \quad N \rightarrow \infty
$$

where $F_{N}(y)$ is defined in (3.28). It is easily demonstrated, see for example Hanna and Rowland (1990), that this is equivalent to proving that

$$
\sum_{n=0}^{\infty} A_{n}^{2} E_{n}=\int_{0}^{a} f^{2}(y) d y+\frac{1}{\alpha_{2}}\left(f^{\prime}(a)\right)^{2}+\frac{1}{\alpha_{1}}\left(f^{\prime}(0)\right)^{2} .
$$

It is clear from (4.5) that

$$
\begin{aligned}
\sum_{n=0}^{N} A_{n}^{2} E_{n} & =\sum_{n=0}^{N} \frac{1}{E_{n}}\left\{\int_{0}^{a} \int_{0}^{a} f(v) f(t) Y_{n}(v) Y_{n}(t) d v d t\right. \\
& +2 \frac{f^{\prime}(a)}{\alpha_{2}} \int_{0}^{a} f(v) Y_{n}(v) Y_{n}^{\prime}(a) d v+2 \frac{f^{\prime}(0)}{\alpha_{1}} \int_{0}^{a} f(v) Y_{n}(v) Y_{n}^{\prime}(0) d v \\
& \left.+\left(\frac{f^{\prime}(a)}{\alpha_{2}} Y_{n}^{\prime}(a)\right)^{2}+\left(\frac{f^{\prime}(0)}{\alpha_{1}} Y_{n}^{\prime}(0)\right)^{2}+2 \frac{f^{\prime}(0) f^{\prime}(a)}{\alpha_{1} \alpha_{2}} Y_{n}^{\prime}(0) Y_{n}^{\prime}(a)\right\}
\end{aligned}
$$

On interchanging the orders of summation and integration, letting $N \rightarrow \infty$ and then using Lemma 1 , it is found that

$$
\begin{aligned}
\sum_{n=0}^{\infty} A_{n}^{2} E_{n} & =\int_{0}^{a} f(v) \int_{-\infty}^{\infty} \mathcal{F}(t)[\delta(v-t)+\delta(-v-t)+\delta(y+t-2 a)] d t d v \\
& +2 \frac{f^{\prime}(a)}{\alpha_{2}} \int_{0}^{a} f(v) \sum_{n=0}^{\infty} \frac{Y_{n}(v) Y_{n}^{\prime}(a)}{E_{n}} d v+2 \frac{f^{\prime}(0)}{\alpha_{1}} \int_{0}^{a} f(v) \sum_{n=0}^{\infty} \frac{Y_{n}(v) Y_{n}^{\prime}(0)}{E_{n}} d v \\
& +\frac{f^{\prime}(0) f^{\prime}(a)}{\alpha_{1} \alpha_{2}} \sum_{n=0}^{\infty} \frac{Y_{n}^{\prime}(0) Y_{n}^{\prime}(a)}{E_{n}} \\
& +\left(\frac{f^{\prime}(a)}{\alpha_{2}}\right)^{2} \sum_{n=0}^{\infty} \frac{\left[Y_{n}^{\prime}(a)\right]^{2}}{E_{n}}+\left(\frac{f^{\prime}(0)}{\alpha_{1}}\right)^{2} \sum_{n=0}^{\infty} \frac{\left[Y_{n}^{\prime}(0)\right]^{2}}{E_{n}}
\end{aligned}
$$

where $\mathcal{F}(t)$ is defined in (3.33). On rearranging the first sum on the right hand side of (4.11) using (2.7) and the second by noting that $Y_{n}^{\prime}(0)=-Q_{0}$, these terms are seen, from (3.1)and 
(3.2), to be zero . The remaining sums are given in (B.1) and (B.2) respectively. It follows that the above expression reduces to (4.9) which confirms that this set of eigenfunctions is indeed complete.

Hence, for this particular example, it is known that the eigenfunctions are complete but linearly dependent and the eigenfunction expansion (3.27), with $A_{n}$ given by (4.5), converges point-wise. As discussed above, the fact that the eigenfunctions are linearly dependent is highly significant to this class of physical problem. Suppose that the waveguide under consideration is semi-infinite, lying in the region $x \geq 0$, see figure 1 . Suppose further that the waveguide is closed by a rigid surface occupying the region $x=0,0 \leq y \leq a$ and that the fundamental fluid-membrane coupled wave is incident, in the negative $x$ direction, through the waveguide towards $x=0$. It is immediately apparent that a possible form for the fluid velocity potential is

$$
\phi(x, y)=Y_{0}(y)\left(e^{i s_{0} x}+e^{-i s_{0} x}\right)
$$

from which it follows that

$$
\phi_{x}(x, y)=i s_{0} Y_{0}(y)\left(e^{i s_{0} x}-e^{-i s_{0} x}\right)
$$

where the subscript indicates differentiation with respect to $x$. This form satisfies all the physical conditions of the problem except the membrane edge conditions. The usual edge conditions enforce either zero membrane displacement or zero gradient at the points $(0,0)$ and $(0, a)$ respectively. Thus, two are required (one for each membrane), consistent with the fact that $K_{0}+K_{a}=2$ for this problem. These conditions can be accommodated by adding a linear combination of the sums (3.1) and (3.2) (both with $q=0$ ) to (4.13). Thus, the velocity $\phi_{x}(x, y)$ can be written as

$$
\phi_{x}(x, y)=i s_{0} Y_{0}(y)\left(e^{i s_{0} x}-e^{-i s_{0} x}\right)+\eta \sum_{n=0}^{\infty} \frac{Y_{n}(y) Y_{n}^{\prime}(a) e^{i s_{n} x}}{\alpha_{2} E_{n}}+\zeta \sum_{n=0}^{\infty} \frac{Y_{n}(y) e^{i s_{n} x}}{E_{n}} .
$$

where $\eta$ where $\zeta$ are arbitrary constants. Note that to obtain this expression (3.1), with $q=0$, has been rearranged using (2.7). On integrating (4.14) with respect to $x$, it is found that

$$
\phi(x, y)=Y_{0}(y)\left(e^{i s_{0} x}+e^{-i s_{0} x}\right)-i \eta \sum_{n=0}^{\infty} \frac{Y_{n}(y) Y_{n}^{\prime}(a) e^{i s_{n} x}}{\alpha_{2} s_{n} E_{n}}-i \zeta \sum_{n=0}^{\infty} \frac{Y_{n}(y) e^{i s_{n} x}}{s_{n} E_{n}} .
$$

The unique solution is obtained once the constants $\eta$ and $\zeta$ are determined via the application of the edge conditions. 


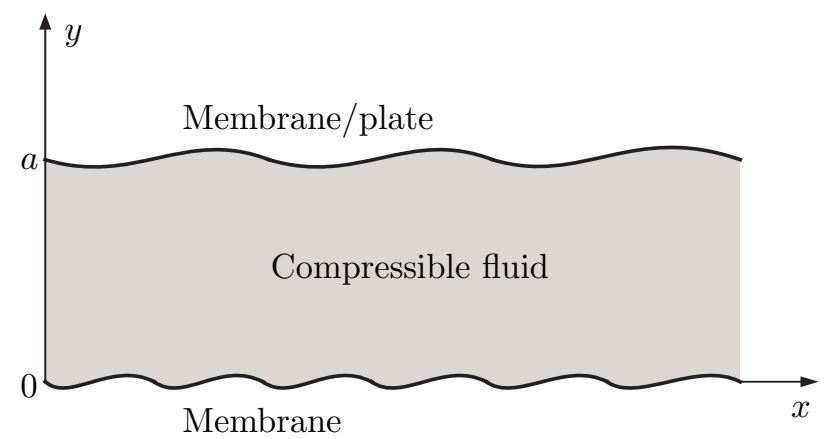

Figure 1: Duct geometry for Examples 1 and 2.

\subsection{Example 2}

The second example, is the case in which the upper duct surface comprises an elastic plate whilst the lower surface is again a membrane. Thus, $P_{0}(s)=-s^{2}+\mu^{2}, Q_{0}=-\alpha$, $P_{a}(s)=s^{4}-\nu^{4}$ and $Q_{a}=-\beta$ where the definitions of the constants $\nu$ and $\beta$ are given (as $k_{0}$ and $\nu$ respectively) by Andronov and Belinsky (1990). In this case the polynomials $\tau(s)$ and $\lambda(s)$, of the dispersion relation, are of higher order than those for Example 1 and complex roots do exist. Again, (4.3) holds but with

$$
\begin{aligned}
\left(Y_{m}, Y_{m}\right) & =\int_{0}^{a} Y_{m} Y_{n} d y+\frac{1}{\beta}\left(Y_{m}^{\prime \prime \prime}(a) Y_{n}^{\prime}(a)+Y_{m}^{\prime}(a) Y_{n}^{\prime \prime \prime}(a)\right) \\
& +\frac{2}{\beta} Y_{m}^{\prime}(a) Y_{n}^{\prime}(a)+\frac{1}{\alpha} Y_{m}^{\prime}(0) Y_{n}^{\prime}(0)
\end{aligned}
$$

As for Example 1, various forms of this orthogonality property have appeared in the literature, for example Andronov and Belinsky (1990), Lawrie and Abrahams (2002), Evans and Porter (2003) and Linton and Chung (2003). Expression (4.16) is not, however, a true inner product since, although the norm can be defined in the same way as for Example 1, that is $\|f\|^{2}=(f, f)$, it cannot be proved, in general, that $\|f\|^{2} \geq 0$. It is nevertheless possible to prove that this set of eigenfunctions is complete, for example, for the class of three times differentiable functions $f(y)$ for which $f^{\prime \prime \prime}(a)=0$. Despite the fact that, for the general case, completeness cannot be proved using the method outlined above, the eigenfunctions for this example satisfy all the other properties discussed in the article.

Suppose that, as for Example 1, the waveguide under consideration is semi-infinite, lying in the region $x \geq 0$ (see figure 1 ), and that that the waveguide is closed by a end plate occupying the region $x=0,0 \leq y \leq a$. In this case, however, forcing is introduced as a specified velocity distribution on the end plate rather than as an incident wave. That 
is, $\phi_{x}(0, y)=f(y), 0 \leq y \leq a$ where $f(y)$ is suitably smooth, for example $f(y)=y^{2}$. (Note that, for the purposes of this example, $f(y) \neq Y_{n}(y), n=0,1,2, \ldots$ since this corresponds to a fluid coupled structural wave for which the problem is tractable via Fourier integrals.) Three edges conditions are required. Without loss of generality, it will be assumed that

$$
\phi_{y}(0,0)=\phi_{y}(0, a)=\phi_{x y}(0, a)=0
$$

which implies zero displacement at both the plate and membrane edges together with zero gradient for the plate. It is convenient to express the normal velocity as an eigenfunction expansion, thus

$$
\phi_{x}(x, y)=\sum_{n=0}^{\infty} A_{n} Y_{n}(y) e^{i s_{n} x} .
$$

It follows from (4.5) and (4.16) that

$$
\begin{aligned}
A_{n} E_{n}=\int_{0}^{a} Y_{n}(y) \phi_{x}(0, y) d y & +\frac{Y_{n}^{\prime \prime \prime}(a)}{\beta} \phi_{x y}(0, a)+\frac{Y_{n}^{\prime}(a)}{\beta} \phi_{x y y y}(0, a) \\
& +\frac{2 Y_{n}^{\prime}(a)}{\beta} \phi_{x y}(0, a)+\frac{Y_{n}^{\prime}(0)}{\alpha} \phi_{x y}(0,0) .
\end{aligned}
$$

On using the condition $\phi_{x y}(0, a)=0$ and denoting $\eta=\phi_{x y y y}(0, a), \zeta=\phi_{x y}(0,0)$, it is found that

$$
A_{n} E_{n}=B_{n}+\frac{\eta}{\beta} Y_{n}^{\prime}(a)+\frac{\zeta}{\alpha} Y_{n}^{\prime}(0)
$$

where

$$
B_{n}=\int_{0}^{a} f(y) Y_{n}(y) d y .
$$

It follows that the velocity potential is given by

$$
\begin{aligned}
\phi(x, y) & =-i \sum_{n=0}^{\infty} \frac{B_{n} Y_{n}(y) e^{i s_{n} x}}{s_{n} E_{n}} \\
& -\frac{i \eta}{\beta} \sum_{n=0}^{\infty} \frac{Y_{n}(y) Y_{n}^{\prime}(a) e^{i s_{n} x}}{s_{n} E_{n}}-i \zeta \sum_{n=0}^{\infty} \frac{Y_{n}(y) e^{i s_{n} x}}{s_{n} E_{n}}
\end{aligned}
$$

where use has been made of the fact that, for this example, $Y_{n}^{\prime}(0)=-Q_{0}=\alpha$. The solution contains two arbitrary constants and, since one edge condition has already been applied, there are two remaining edge conditions by which to determine them. Note that, for this example, $K_{a}+K_{0}=3$ which, as expected, is the same as the total number of edge conditions. It is clear that, even though completeness has not been established for this set of eigenfunctions, the velocity potential has been expressed as an eigenfunction expansion which, according to Theorem 2, converges point-wise to the solution. 


\section{Concluding remarks}

In this article a class of expansion involving the eigenfunctions described by equations (2.6)(2.8) has been investigated. Attention has been restricted (see conditions i), ii) and iii) of section 2) to the cases $Q_{0(a)}=0, P_{0(a)} \neq 0$ with $\left|P_{a(0)}(s) / Q_{a(0)}(s)\right|=O\left(s^{2\left(K_{a(0)}-J_{a(0)}\right)}\right)$ as $|s| \rightarrow \infty$ or $\left|P_{p}(s) / Q_{p}(s)\right|=O\left(s^{2\left(K_{p}-J_{p}\right)}\right)$ as $|s| \rightarrow \infty$ for both $p=a$ and $p=0$, where $K_{p}-J_{p}>0$ is a positive integer. This restriction ensures that the eigenfunction expansions discussed herein are a generalisation of the Fourier cosine series in that the eigenvalues are given, to leading order by, $\gamma_{n+K_{a}+K_{0}} \sim i n \pi / a$ as $n \rightarrow \infty$, see (2.20). Many real physical boundary conditions, for example those describing membranes and elastic plates, do in fact satisfy this constraint. It has been proved that the eigenfunctions are linearly dependent and that the number of "excess" eigenfunctions is the same as the number of edge conditions to be applied for a given problem. Most significantly, it has been proved that the eigenfunction expansions given by (3.27) converge point-wise. Completeness of the eigenfunctions has been proved for one specific example and has been discussed for a second case. It remains, however, to prove completeness for the general case.

\section{A The orthogonality relation}

The eigensystem for the functions $Y_{n}(y)$ is specified in equations (2.6)-(2.8). In the analysis that follows it is convenient to use the function $Y(s, y)$ defined by (3.4). Recall that $Y_{n}(y)=Y\left(s_{n}, y\right)$ where $s_{n}, n=0,1,2, \ldots$ are the roots to $(2.10)$, that is $K(s)=0$ where

$$
K(s)=P_{a}(s) Y^{\prime}(s, a)+Q_{a}(s) Y(s, a) .
$$

Note that the prime refers to differentiation with respect to $y$. When differentiation with respect to $s$ is required this will be written explicitly.

From (2.7)-(2.8) it is clear that

$$
\begin{gathered}
{\left[\left\{P_{a}\left(s_{n}\right) Y_{n}^{\prime}(y)+Q_{a}\left(s_{n}\right) Y_{n}(y)\right\}\left\{P_{0}\left(s_{m}\right) Y_{m}^{\prime}(y)+Q_{0}\left(s_{m}\right) Y_{m}(y)\right\}\right.} \\
\left.-\left\{P_{a}\left(s_{m}\right) Y_{m}^{\prime}(y)+Q_{a}\left(s_{m}\right) Y_{m}(y)\right\}\left\{P_{0}\left(s_{n}\right) Y_{n}^{\prime}(y)+Q_{0}\left(s_{n}\right) Y_{n}(y)\right\}\right]_{0}^{a}=0 .
\end{gathered}
$$

On expanding and rearranging this it is found that

$$
\left[t_{m n} Y_{n}(y) Y_{m}^{\prime}(y)-t_{n m} Y_{m}(y) Y_{n}^{\prime}(y)+\left(\gamma_{m}^{2}-\gamma_{n}^{2}\right)\left\{p_{m n} Y_{m}^{\prime}(y) Y_{n}^{\prime}(y)+q_{m n} Y_{m}(y) Y_{n}(y)\right\}\right]_{0}^{a}=0
$$


where $t_{m n}, p_{m n}$ and $q_{m n}$ are defined in equations (2.12)-(2.14). It is now an easy matter to write the first term of (A.3) in integral form to obtain

$$
\begin{aligned}
t_{m n} \int_{0}^{a}\left(Y_{n}^{\prime}(y) Y_{m}^{\prime}(y)+Y_{n}(y) Y_{m}^{\prime \prime}(y)\right) d y & -t_{n m}\left[Y_{m}(y) Y_{n}^{\prime}(y)\right]_{0}^{a} \\
+\left(\gamma_{m}^{2}-\gamma_{n}^{2}\right)\left[p_{m n} Y_{m}^{\prime}(y) Y_{n}^{\prime}(y)\right. & \left.+q_{m n} Y_{m}(y) Y_{n}(y)\right]_{0}^{a}=0
\end{aligned}
$$

Then, on integrating the first term of the integral by parts, it is found that

$$
\begin{aligned}
\left(\gamma_{m}^{2}-\gamma_{n}^{2}\right)\left\{t_{m n}\right. & \int_{0}^{a} Y_{m}(y) Y_{n}(y) d y+r_{m n}\left[Y_{m}(y) Y_{n}^{\prime}(y)\right]_{0}^{a} \\
& \left.+\left[p_{m n} Y_{m}^{\prime}(y) Y_{n}^{\prime}(y)+q_{m n} Y_{m}(y) Y_{n}(y)\right]_{0}^{a}\right\}=0
\end{aligned}
$$

where $r_{m n}$ is given by (2.15). Thus, the expression within the parentheses is equal to zero provided $m \neq n$. It remains to consider the case $m=n$. On dividing (A.2) and (A.5) by $s_{m}^{2}-s_{n}^{2}$ and equating the resulting expressions, it is found that

$$
\begin{aligned}
t_{n n} \int_{0}^{a} Y_{n}^{2} d y & +p_{n n}\left(\left[Y_{n}^{\prime}(a)\right]^{2}-\left[Y_{n}^{\prime}(0)\right]^{2}\right)+q_{n n}\left(Y_{n}^{2}(a)-Y_{n}^{2}(0)\right) \\
& +r_{n n}\left(Y_{n}(a) Y_{n}^{\prime}(a)-Y_{n}(0) Y_{n}^{\prime}(0)\right) \\
& =\left[\left\{P_{a}\left(s_{n}\right) Y_{n}^{\prime}(y)+Q_{a}\left(s_{n}\right) Y_{n}(y)\right\} \lim _{s \rightarrow s_{n}} \frac{P_{0}(s) Y^{\prime}(s, y)+Q_{0}(s) Y(s, y)}{\left(s^{2}-s_{n}^{2}\right)}\right. \\
& \left.-\lim _{s \rightarrow s_{n}} \frac{P_{a}(s) Y^{\prime}(s, y)+Q_{a}(s) Y(s, y)}{\left(s^{2}-s_{n}^{2}\right)}\left\{P_{0}\left(s_{n}\right) Y_{n}^{\prime}(y)+Q_{0}\left(s_{n}\right) Y_{n}(y)\right\}\right]_{0}^{a} .
\end{aligned}
$$

On using L'Hôpital's rule to evaluate the limit on the right hand side and inserting the values $y=a, 0$, expression (A.6) reduces to

$$
\begin{aligned}
t_{n n} \int_{0}^{a} Y_{n}^{2} d y & +p_{n n}\left(\left[Y_{n}^{\prime}(a)\right]^{2}-\left[Y_{n}^{\prime}(0)\right]^{2}\right)+q_{n n}\left(Y_{n}^{2}(a)-Y_{n}^{2}(0)\right) \\
& +r_{n n}\left(Y_{n}(a) Y_{n}^{\prime}(a)-Y_{n}(0) Y_{n}^{\prime}(0)\right) \\
& =-\left.\frac{\left\{P_{0}\left(s_{n}\right) Y_{n}^{\prime}(a)+Q_{0}\left(s_{n}\right) Y_{n}(a)\right\}}{2 s_{n}} \frac{d}{d s} K(s)\right|_{s=s_{n}}=t_{n n} E_{n} .
\end{aligned}
$$

Hence, (A.2) may be written as

$$
\begin{aligned}
t_{m n} \int_{0}^{a} Y_{m} Y_{n} d y & +p_{m n}\left(Y_{m}^{\prime}(a) Y_{n}^{\prime}(a)-Y_{m}^{\prime}(0) Y_{n}^{\prime}(0)\right) \\
& +q_{m n}\left(Y_{m}(a) Y_{n}(a)-Y_{m}(0) Y_{n}(0)\right) \\
& +r_{m n}\left(Y_{m}(a) Y_{n}^{\prime}(a)-Y_{m}(0) Y_{n}^{\prime}(0)\right)=\delta_{m n} t_{m n} E_{n}
\end{aligned}
$$

Expression (2.11) follows on interchanging $m$ and $n$ in (A.8) and adding the result to (A.8). 


\section{B Evaluation of some relevant sums}

The following sums are used in the discussion

$$
\sum_{n=0}^{\infty} \frac{Y_{n}^{\prime}(y) Y_{n}^{\prime}(a) \gamma_{n}^{2 q}}{E_{n}}= \begin{cases}0, & 0 \leq y<a \\ C, & y=a\end{cases}
$$

and

$$
\sum_{n=0}^{\infty} \frac{\left[Y_{n}^{\prime}(0)\right]^{2} \gamma_{n}^{2 r}}{E_{n}}=D
$$

where $q=0,1,2, \ldots K_{a}-J_{a}-1, r=0,1,2, \ldots K_{0}-J_{0}-1$ and

$$
C=\lim _{|s| \rightarrow \infty}\left|\frac{Q_{a}(s) s^{2 q+2}}{P_{a}(s)}\right| \quad \text { and } \quad D=\lim _{|s| \rightarrow \infty}\left|\frac{Q_{0}(s) s^{2 r+2}}{P_{0}(s)}\right| .
$$

These sums may be evaluated in the same way as those demonstrated in section 3 . The appropriate integrals are:

$$
I_{6}(y)=\frac{1}{2 \pi i} \int_{-\infty}^{\infty} \frac{s Y^{\prime}(s, y) Q_{a}(s) \gamma^{2 q}}{K(s)} d s, \quad 0 \leq y \leq a
$$

and

$$
I_{7}(y)=\frac{1}{2 \pi i} \int_{-\infty}^{\infty} \frac{s\left[Y^{\prime}(s, 0)\right]^{2} P_{a}(s) \gamma^{2 r}}{K(s) Y(s, a)} d s
$$

\section{References}

[1] Andronov, I.V. and Belinsky, B.P. (1990) "On acoustic boundary-contact problems for a vertically stratifed medium bounded from above by a plate with concentrated inhomogeneities" Prikl. Matem. Mekhan., 54, 366-371.

[2] Churchill, R.V., J.W. Brown, JW and Verhey, R.F. (1974) "Complex variables and applications" Third Edition, McGraw Hill, ISBN 070854157.

[3] Evans, D.V. and Porter, R. (2003) "Wave scattering by narrow cracks in ice sheets floating on water of finite depth", J.Fluid Mech. 484, 143-165.

[4] Gradshteyn, I.S. and Rhzhik, I.M. (1980) "Table of integrals, series and products ", Fifth Edition, Academic Press, ISBN 012294755X.

[5] Hanna, J.R. and Rowland,J.H. (1990) "Fourier series, transforms, and boundary value problems" Second Edition, Wiley Interscience, ISBN 0471619833. 
[6] Kaplunov, Y.D., Kirillova, I.V. and Postnova, Y.A. (2004) "Dispersion of waves in a plane acoustic layer with flexible elastic walls." Acoustical Physics, 50, 694-698.

[7] Lawrie, J.B. and Abrahams, I.D. (1999) "An orthogonality condition for a class of problem with high order boundary conditions; applications in sound/structure interaction" Q. Jl Mech appl. Math. 52, 161-181.

[8] Lawrie, J.B. and Abrahams, I.D. (2002) "On the propagation and scattering of fluidstructural waves in a three-dimensional duct bounded by thin elastic walls." Proceedings of IUTAM 2000/10, Kluwer. Eds. I.D. Abrahams et. al. ISBN 1-4020-0590-3.

[9] Lawrie, J.B. and Guled, I.M.M. (2006) "On tuning a reactive silencer by vaying the position of an internal membrane." Jl Acoust Soc Am, 120, 780-790.

[10] Lawrie, J.B. and Kirby, R. (2006) "Mode-matching without root-finding: application to a dissipative silencer." Jl Acoust Soc Am, 119, 2050-2061.

[11] Linton, C.M. and Chung, H. (2003) "Reflection and transmission at the ocean/sea-ice boundary" Wave Motion, 38(1), 43-52.

[12] Noble, B. (1988) "Methods based on the Wiener-Hopf Technique" Second Edition, Chelsea Publishing Company, New York.

[13] Warren, D.P., Lawrie, J.B. and Mohamed, I.M. (2002) "Acoustic scattering in waveguides with discontinuites in height and material property" Wave Motion, 36, 119-142.

\section{Errata:}

$$
\begin{aligned}
2 \sum_{n=0}^{\infty} \frac{d_{n} P_{0}\left(d_{n}\right) \sinh \left[\delta_{n}(y-a)\right] \cosh \left[\delta_{n}(v-a)\right]}{\left.\left(d_{n}^{2}-1\right) \sinh \left(\delta_{n} a\right) \frac{d}{d s} Y(s, a)\right|_{s=d_{n}}} & =-2 H(-v-y)+\frac{P_{0}(1)(a-y)}{a\left\{P_{0}(1)-a Q_{0}(1)\right\}} \\
& -\frac{2}{\pi} \sum_{n=1}^{\infty} \frac{1}{n} \sin \left(\frac{n \pi y}{a}\right) \cos \left(\frac{n \pi v}{a}\right) \\
\sum_{n=0}^{\infty} \frac{Y_{n}(y) Z_{n}(v)}{E_{n}}= & H(v-y)-H(-v-y)+H(v+y-2 a)-\left(1-\frac{y}{a}\right) \\
+ & \frac{Q_{0}(1) P_{0}(1)\left\{P_{0}(1)-y Q_{0}(1)\right\}}{K(1)\left\{P_{0}(1)-a Q_{0}(1)\right\}}+\frac{P_{0}(1)(a-y)}{a\left\{P_{0}(1)-a Q_{0}(1)\right\}}
\end{aligned}
$$

